OPEN ACCESS

Edited by:

Lixin Shen,

Syracuse University, USA

Reviewed by:

Jian Lu,

Shenzhen University, China

Xiaosheng Zhuang,

City University of Hong Kong,

Hong Kong

*Correspondence:

Jürgen Prestin

prestin@math.uni-luebeck.de

Specialty section:

This article was submitted to Mathematics of Computation and

Data Science,

a section of the journal

Frontiers in Applied Mathematics and

Statistics

Received: 21 December 2016 Accepted: 13 March 2017

Published: 28 March 2017

Citation:

Derevianko N, Myroniuk V and

Prestin J (2017) Characterization of Local Besov Spaces via Wavelet Basis

Expansions.

Front. Appl. Math. Stat. 3:4. doi: 10.3389/fams.2017.00004

\section{Characterization of Local Besov Spaces via Wavelet Basis Expansions}

\author{
Nadiia Derevianko $^{1}$, Vitalii Myroniuk ${ }^{1}$ and Jürgen Prestin ${ }^{2 *}$ \\ ${ }^{1}$ Department of Theory of Functions, Institute of Mathematics of NAS of Ukraine, Kyiv, Ukraine, ${ }^{2}$ Institut für Mathematik, \\ Universität zu Lübeck, Lübeck, Germany
}

In this paper we deal with local Besov spaces of periodic functions of one variable. We characterize these spaces in terms of summability conditions on the coefficients in series expansions of their elements with respect to an orthogonal Schauder basis of trigonometric polynomials. We consider a Schauder basis that was constructed by using ideas of a periodic multiresolution analysis and corresponding wavelet spaces. As an interim result we obtain a characterization of local Besov spaces via operators of the orthogonal projection on the corresponding scaling and wavelet spaces. In order to achieve our new results, we substantially use a theorem on the discretization of scaling and wavelet spaces as well as a connection between local and usual classical Besov spaces. The corresponding characterizations are also given for the classical Besov spaces.

Keywords: local Besov spaces, Schauder basis, Fourier coefficients, periodic multiresolution analysis, wavelets, scaling functions, trigonometric polynomials 2010 Mathematics Subject Classification: 42A10, 42C40.

\section{INTRODUCTION}

One of the crucial problems in the theory of approximation is to describe the smoothness properties of functions by the behavior of the coefficients in their series expansions in terms of given bases or frames. Besov spaces and their generalizations are particularly suitable for such studies. Recent papers describing the smoothness of functions from these spaces by the decay of the coefficient sequences are e.g., Bazarkhanov [1] for Meyer wavelets, Dinh [2] for mixed B-splines, and Hinrichs et al. [3] for Faber-Schauder bases.

In the present paper we consider this problem for local Besov spaces of periodic functions of one variable with respect to some orthogonal trigonometric Schauder basis. Let us first give some motivation of our work. Let

$$
\sum_{n=-\infty}^{\infty} a_{n}(f) \mathrm{e}^{\mathrm{i} n x}
$$

be the Fourier series of some function $f \in L_{2 \pi}^{2}, a_{n}(f)$ the Fourier coefficients of $f$.

In view of the Parseval's equality, it is easy to obtain the following result about the description of the usual classical Besov spaces $B_{2, \theta}^{\alpha}$ of periodic functions: $f \in B_{2, \theta}^{\alpha}, \alpha>0$, if and only if $f \in L_{2 \pi}^{2}$ and the norm 


$$
\left\{\begin{array}{l}
\left(\left|a_{0}(f)\right|^{\theta}+\sum_{n=1}^{\infty}\left[2^{n \alpha}\left(\sum_{2^{n-1} \leq|k|<2^{n}}\left|a_{k}(f)\right|^{2}\right)^{1 / 2}\right]^{\theta}\right)^{1 / \theta} \\
1 \leq \theta<\infty \\
\sup _{n \in \mathbb{N}}\left(\left|a_{0}(f)\right|+2^{n \alpha}\left(\sum_{2^{n-1} \leq|k|<2^{n}}\left|a_{k}(f)\right|^{2}\right)^{1 / 2}\right) \\
\theta=\infty
\end{array}\right.
$$

is finite.

On the other hand, local properties of functions from the Besov spaces can be investigated by expanding them in a series with respect to the Haar basis. To give a short description of these results let for $n \in \mathbb{N}, v(n)=\left\{k: k=0, \ldots, 2^{n-1}-1\right\}, v(0)=\{0\}$ be the sets of indices and $\left\{h_{n, k}\right\}, n \in \mathbb{N}_{0}, k \in v(n)$ be the Haar system. This system is an orthogonal Schauder basis in the space $L^{p}([0,1]), 1 \leq p<\infty$, and for every $f \in L^{p}([0,1])$

$$
f(x)=\sum_{n=0}^{\infty} \sum_{k \in v(n)} b_{n, k}(f) h_{n, k}(x)
$$

in the sense of the norm of $L^{p}([0,1])$ [4, Chap. 3].

Romanyuk [5] obtained necessary and sufficient conditions on the Fourier-Haar coefficients $b_{n, k}(f)$ at which functions from $L^{p}([0,1])$ belong to the Besov spaces. Namely, let $1 \leq p, \theta<\infty$, $0<\alpha<1 / p$, then $f \in B_{p, \theta}^{\alpha}$ if and only if $f \in L^{p}([0,1])$ and the norm

$$
\left(\sum_{n=0}^{\infty}\left[2^{n(\alpha-1 / p+1 / 2)}\left(\sum_{k \in v(n)}\left|b_{n, k}(f)\right|^{p}\right)^{1 / p}\right]^{\theta}\right)^{1 / \theta}
$$

is finite. In this case it is evident that the Fourier-Haar coefficients $b_{n, k}(f)$ describe the local behavior of the function $f$. Note that $\mathrm{V}$. Romanyuk considered the multivariate case, but since in the present paper we investigate functions of one variable, we formulate his result only in the univariate case.

Our aim in this paper is to combine these two approaches and to describe local smoothness of periodic functions in terms of summability conditions on the Fourier coefficients with respect to an orthogonal Schauder basis of trigonometric polynomials in the space $L_{2 \pi}^{p}$ for all $1 \leq p \leq \infty$. The local smoothness is understood in the sense of Besov spaces. We call these Besovtype spaces as local Besov spaces (see Subsection 2.1 for a definition).

Note that some results in this direction were obtained by Mhaskar and Prestin [6]. There expansions of functions from the local Besov spaces in series with respect to a system of trigonometric frames were considered and these spaces were described via coefficients of these expansions. However, this system is not a Schauder basis.

Let us sketch the main results of the present paper. Let $\psi_{0}$ be a scaling function of a periodic multiresolution analysis (PMRA) generated by de la Vallée Poussin means and $\psi_{n}, n \in \mathbb{N}$, be corresponding wavelets [7]. Let $N_{n}:=3 \cdot 2^{n}$ for $n \in \mathbb{N}$ and $N_{0}:=N_{1}$. By $\psi_{n, s}$ we denote shifts of $\psi_{n}$ :

$$
\psi_{n, s}(\cdot):=\psi_{n}\left(\cdot-\frac{s \pi}{N_{n}}\right), \quad n \in \mathbb{N}_{0}, s=0, \ldots, 2 N_{n}-1 .
$$

We show that for a particular choice of $\psi_{n}$ the system $\left\{\psi_{n, s}\right\}$ constitutes an orthogonal trigonometric Schauder basis in the space $L_{2 \pi}^{p}, 1 \leq p \leq \infty$, and a function $f \in L_{2 \pi}^{p}$ can be represented by a series (for more detailed information see Subsection 2.2)

$$
f(x)=\sum_{n=0}^{\infty} \sum_{s=0}^{2 N_{n}-1} a_{n, s}(f) \psi_{n, s}(x), \quad x \in \mathbb{R}
$$

converging in the norm of the space $L_{2 \pi}^{p}$, where the coefficient functionals $a_{n, s}(f)$ are Fourier coefficients of $f$ with respect to the basis $\left\{\psi_{n, s}\right\}$ :

$$
a_{n, s}(f)=\left\langle f, \psi_{n, s}\right\rangle=\frac{1}{2 \pi} \int_{0}^{2 \pi} f(x) \psi_{n, s}(x) \mathrm{d} x .
$$

Because $\psi_{n}$ is even (see Subsection 2.2 for definition) and from (1.3) we conclude that $a_{n, s}(f)$ can be represented in the following way:

$$
a_{n, s}(f)=\frac{1}{2 \pi} \int_{0}^{2 \pi} f(x) \psi_{n}\left(\frac{s \pi}{N_{n}}-x\right) \mathrm{d} x=\left(f * \psi_{n}\right)\left(\frac{s \pi}{N_{n}}\right),
$$

where $f * g$ means the convolution

$$
(f * g)(x):=\frac{1}{2 \pi} \int_{0}^{2 \pi} f(y) g(x-y) \mathrm{d} y, \quad x \in \mathbb{R} .
$$

For more information regarding trigonometric Schauder bases we refer to Lorentz and Sakyan [8], Prestin and Selig [7], Selig [9] and the references cited there.

Let $I \subset \mathbb{R},|I|<2 \pi$, be some segment and $n \in \mathbb{N}$. By $\kappa(I, n)$ we denote the set of indices $s$ which satisfy the properties $s=0, \ldots, 2 N_{n}-1$ and there exists $k \in \mathbb{Z}$ such that the point $\frac{\left(s+2 k N_{n}\right) \pi}{N_{n}}$ belongs to the segment $I$. For $1 \leq p \leq \infty, n \in \mathbb{N}_{0}$, and the segment $I$ we define the following sequence

$$
c_{n}(I, p):= \begin{cases}N_{n}^{1 / 2-1 / p}\left(\sum_{s \in \kappa(I, n)}\left|a_{n, s}(f)\right|^{p}\right)^{1 / p}, & 1 \leq p<\infty, \\ N_{n}^{1 / 2} \max _{s \in \kappa(I, n)}\left|a_{n, s}(f)\right|, & p=\infty .\end{cases}
$$

Let $1 \leq p \leq \infty, f \in L_{2 \pi}^{p}, x_{0} \in[0,2 \pi), 0<\alpha<1$, $0<\theta \leq \infty$. Then the main result of this paper is written as follows: A function $f$ belongs to the local Besov spaces $B_{p, \theta}^{\alpha}\left(x_{0}\right)$ if and only if there exists an interval $I \subset \mathbb{R},|I|<2 \pi$, centered at $x_{0}$, such that the norm 


$$
\begin{cases}\left(\sum_{n=0}^{\infty} 2^{n \theta \alpha}\left|c_{n}(I, p)\right|^{\theta}\right)^{1 / \theta}, & 0<\theta<\infty \\ \sup _{n \in \mathbb{N}_{0}} 2^{n \alpha}\left|c_{n}(I, p)\right|, & \theta=\infty\end{cases}
$$

is finite.

We adopt the following convention regarding constants. The letters $C, C_{i}, i=1,2, \ldots$, mean positive constants which may depend on parameters fixed for the spaces. Their values are not necessarily the same in different parts of the text. When constants depend on functions $f, \zeta$ or some intervals $I$, we indicate this in brackets.

The present paper is organized as follows: In Subsection 2.1 we define the local Besov spaces and formulate their connection with classical Besov spaces. In Subsection 2.2 we give definitions of the orthogonal trigonometric Schauder basis that we work with and describe expansions of functions from $L_{2 \pi}^{p}$ in a series with respect to this basis. In Section 3 we formulate the main results of this paper. In Section 4 we prove the main auxiliary statements. In Section 5 we give proofs of the main results of the present paper.

\section{PRELIMINARIES}

As stated in Section 1, our aim in this paper is to describe the local Besov spaces of functions $f$ in terms of summability conditions on the coefficients in a series expansion of $f$ as in (1.4).

Let us first agree about the notation. As usual $\mathbb{N}$ is reserved for the natural numbers, by $\mathbb{N}_{0}$ we denote the natural numbers including 0 , by $\mathbb{Z}$ the set of all integers and by $\mathbb{R}$ the set of all real numbers.

Let $1 \leq p \leq \infty$ and $A \subset \mathbb{R}$ be a Lebesgue measurable set. By $L^{p}(A)$ we denote the space of functions $f: A \rightarrow \mathbb{R}$ Lebesgue measurable on $A$ with the finite norm

$$
\|f\|_{A, p}:= \begin{cases}\left(\int_{A}|f(t)|^{p} \mathrm{~d} t\right)^{1 / p}, & 1 \leq p<\infty, \\ \underset{t \in A}{\operatorname{ess} \sup }|f(t)|, & p=\infty .\end{cases}
$$

When $A=[0,2 \pi)$, we understand by $L^{p}([0,2 \pi))$ the space of functions $f$ defined on the segment $[0,2 \pi)$ and extended $2 \pi$-periodically to the real line with natural modification for the norm $\|f\|_{[0,2 \pi), p}:=\left(\frac{1}{2 \pi} \int_{0}^{2 \pi}|f(t)|^{p} \mathrm{~d} t\right)^{1 / p}$. For simplicity, by $L^{\infty}([0,2 \pi))$ we denote the space of $2 \pi$-periodic continuous functions (equipped with $\|f\|_{[0,2 \pi), \infty}$ as its norm). Further, we will write $L_{2 \pi}^{p}$ instead of $L^{p}([0,2 \pi))$ and $\|f\|_{p}$ instead of $\|f\|_{[0,2 \pi), p}$.

\subsection{The Local Besov Spaces of Functions and Their Connection with Classical Besov Spaces}

Let us introduce the local Besov spaces $B_{p, \theta}^{\alpha}\left(x_{0}\right)$ of periodic functions. For a function $f \in L^{p}(I)$ where $I=[a, b] \subseteq \mathbb{R}$ we define the $r$ th difference operator $\Delta_{t}^{r}$ by

$$
\Delta_{t}^{r} f(\cdot)=\sum_{k=0}^{r}(-1)^{r-k}\left(\begin{array}{l}
r \\
k
\end{array}\right) f(\cdot+k t), \quad r \in \mathbb{N}, t \in \mathbb{R},
$$

and for $0<\delta<(b-a) / r$ we define the modulus of smoothness by

$$
\omega_{I, r, p}(f, \delta):=\sup _{0<t \leq \delta}\left\|\Delta_{t}^{r} f(\cdot)\right\|_{[a, b-r \delta], p} .
$$

If $\delta \geq(b-a) / r$, we put

$$
\omega_{I, r, p}(f, \delta):=\inf \|f-P\|_{I, p},
$$

where the infimum is taken over all algebraic polynomials of degree at most $r-1$.

It will be convenient for us to use a sequential version of the Besov spaces which we now define. For a sequence $\mathbf{a}=\left\{a_{n}\right\}_{n=0}^{\infty}$ and numbers $\alpha, \theta>0$ by $\|\mathbf{a}\|_{\theta, \alpha}$ we denote the following norm

$$
\|\mathbf{a}\|_{\theta, \alpha}:= \begin{cases}\left(\sum_{n=0}^{\infty}\left(2^{n \alpha}\left|a_{n}\right|\right)^{\theta}\right)^{1 / \theta}, & 0<\theta<\infty, \\ \sup _{n \in \mathbb{N}_{0}} 2^{n \alpha}\left|a_{n}\right|, & \theta=\infty .\end{cases}
$$

The notation $\mathbf{a} \in b_{\theta, \alpha}$ means that the norm $\|\mathbf{a}\|_{\theta, \alpha}$ is finite.

Let $1 \leq p \leq \infty, 0<\theta \leq \infty, \alpha>0$ and $r=[\alpha]+1$. For $x_{0} \in \mathbb{R}$ the local Besov space $B_{p, \theta}^{\alpha}\left(x_{0}\right)$ is the collection of functions $f$ which satisfy the following properties:

(1) $f \in L_{2 \pi}^{p}$;

(2) there is a non-degenerate interval $I \subset \mathbb{R},|I|<2 \pi$, centered at $x_{0}$, such that

$$
\left\{\omega_{I, r, p}\left(f, 2^{-n}\right)\right\}_{n=0}^{\infty} \in b_{\theta, \alpha} .
$$

The spaces $B_{p, \theta}^{\alpha}\left(x_{0}\right)$ were considered in Mhaskar and Prestin [6]. By periodicity we can restrict ourselves to points $x_{0} \in[0,2 \pi)$.

In order to prove our main results, we use a connection between the local and the classical Besov spaces of periodic functions. Let us define these Besov spaces.

Let $f \in L_{2 \pi}^{p}, 1 \leq p \leq \infty$. For $\delta>0$ we define the modulus of smoothness by

$$
\omega_{r, p}^{*}(f, \delta):=\sup _{0<t \leq \delta}\left\|\Delta_{t}^{r} f(\cdot)\right\|_{p},
$$

where, in contrast to the modulus of smoothness $\omega_{I, r, p}(f, \delta)$, the norm is taken over the entire period of $f$, using the periodicity of $f$ in the case when the translates go outside of $[0,2 \pi)$. Let $1 \leq p \leq \infty, \alpha>0,0<\theta \leq \infty$ and $r$ be some integer number greater than $\alpha$. The classical Besov space $B_{p, \theta}^{\alpha *}$ consists of functions $f$ such that $f \in L_{2 \pi}^{p}$ and the sequence $\left\{\omega_{r, p}^{*}\left(f, 2^{-n}\right)\right\}_{n=0}^{\infty} \in b_{\theta, \alpha}$ for some integer $r>\alpha$. The space $B_{p, \theta}^{\alpha *}$ is independent of the choice of $r$ as long as $r>\alpha$ (see [10, Theorem 10.1, Chapter 2]). One can find more information about Besov spaces in the monographs $[10,11]$. The following statement about the connection between the two spaces mentioned above is proved in the paper [6].

Proposition A. Let $1 \leq p \leq \infty, f \in L_{2 \pi}^{p}, \alpha>0,0<$ $\theta \leq \infty, x_{0} \in[0,2 \pi)$. Then $f \in B_{p, \theta}^{\alpha}\left(x_{0}\right)$ if and only if there exists an interval $I \subset \mathbb{R},|I|<2 \pi$, centered at $x_{0}$ such that for every infinitely differentiable on $\mathbb{R}$ function $\zeta$ supported on $I$ and extended as a $2 \pi$-periodic function, the function $f \zeta$ is in $B_{p, \theta}^{\alpha *}$. 


\subsection{Expansions in a Series}

Let us first give some necessary definitions. Let

$$
g_{1}(x):= \begin{cases}\sqrt{2}, & -\frac{\pi}{2}+\frac{\pi}{6} \leq x \leq \frac{\pi}{2}-\frac{\pi}{6} \\ \frac{1-3\left(\frac{2|x|}{\pi}-1\right)}{\sqrt{1+\left(3\left(\frac{2|x|}{\pi}-1\right)\right)^{2}},} & \frac{\pi}{2}-\frac{\pi}{6} \leq|x| \leq \frac{\pi}{2}+\frac{\pi}{6} \\ 0, & \frac{\pi}{2}+\frac{\pi}{6} \leq|x| \leq \pi\end{cases}
$$

be a coefficient function given on the segment $[-\pi, \pi]$ and let as above $N_{n}=3 \cdot 2^{n}$ and $M_{n}:=2^{n}, n \in \mathbb{N}$. By $\varphi_{n}$ we denote the following function

$$
\varphi_{n}(\cdot):=\frac{1}{2 \sqrt{N_{n}}} \sum_{k=-N_{n}-M_{n}}^{N_{n}+M_{n}} g_{1}\left(\frac{k \pi}{2 N_{n}}\right) \cos k \cdot,
$$

which is a modification of the de la Vallée Poussin kernel.

Let as above $\varphi_{n, s}$ denote shifts of the function $\varphi_{n}$ :

$$
\varphi_{n, s}(\cdot):=\varphi_{n}\left(\cdot-\frac{s \pi}{N_{n}}\right), n \in \mathbb{N}, s=0, \ldots, 2 N_{n}-1 .
$$

In Prestin and Selig [7] it is proved that the sequence of spaces $\left\{V_{n}\right\}_{n=1}^{\infty}$, defined as

$$
V_{n}:=\operatorname{span}\left\{\varphi_{n, s}, s=0, \ldots, 2 N_{n}-1\right\},
$$

provides a PMRA in $L_{2 \pi}^{2}$, i.e., the system $\left\{\varphi_{n, s}, s=0, \ldots, 2 N_{n}-1\right\}$ is a basis in the space $V_{n} ; V_{n} \subset V_{n+1}$ for all $n \in \mathbb{N}$; it holds that $\operatorname{clos}_{L_{2 \pi}^{2}}\left(\bigcup_{n \in \mathbb{N}} V_{n}\right)=L_{2 \pi}^{2}$. The functions $\varphi_{n}$ are called scaling functions of this PMRA and the spaces $V_{n}$ are called scaling spaces.

The wavelet space $W_{n}$ which is defined to be the orthogonal complement of $V_{n}$ with respect to $V_{n+1}$, i.e., $W_{n}=V_{n+1} \ominus V_{n}$, is spanned by the translates of the function $\psi_{n}[7]$ :

$$
W_{n}=\operatorname{span}\left\{\psi_{n, s}, s=0, \ldots, 2 N_{n}-1\right\},
$$

where

$$
\psi_{n}(\cdot)=\frac{1}{\sqrt{N_{n}}} \sum_{k=-M_{n}}^{2 N_{n}-M_{n}} g_{2}\left(\frac{k \pi}{N_{n}}\right) \cos \left(N_{n}+k\right)
$$

and

$$
g_{2}(x)= \begin{cases}\frac{1+\frac{3 x}{\pi}}{\sqrt{1+\left(\frac{3 x}{\pi}\right)^{2}},}, & -\frac{\pi}{3} \leq x \leq \frac{\pi}{3} \\ \frac{2-3\left(\frac{x}{\pi}-1\right)}{\sqrt{4+\left(3\left(\frac{x}{\pi}-1\right)\right)^{2}}}, & \frac{\pi}{3}<x \leq 2 \pi-\frac{\pi}{3}\end{cases}
$$

is a coefficient function given on the segment $\left[-\frac{\pi}{3}, 2 \pi-\frac{\pi}{3}\right]$. The functions $\psi_{n}$ are called wavelets. Note that the functions $g_{1}$ and $g_{2}$ defined on the segments with lengths equal $2 \pi$ can be extended to $\mathbb{R}$ as $2 \pi$-periodic functions (and continuous since $g_{1}(-\pi)=g_{1}(\pi)=0$ and $\left.g_{2}\left(-\frac{\pi}{3}\right)=g_{1}\left(2 \pi-\frac{\pi}{3}\right)=0\right)$.
For simplicity of notations we denote $\psi_{0}:=\varphi_{1}, N_{0}:=N_{1}$ and for $n \in \mathbb{N}_{0}, s=0, \ldots, 2 N_{n}-1$,

$$
t_{2 N_{n}+s}(\cdot):=\psi_{n, s}(\cdot)
$$

For $f \in L_{2 \pi}^{p}, 1 \leq p \leq \infty$, we define some trigonometric polynomial operators $\sigma_{n}$. Let $n \in \mathbb{N}_{0}, x \in \mathbb{R}$ and

$$
\sigma_{n}(f, x):=\sum_{k=0}^{2 N_{n-2}-1}\left\langle f, t_{k}\right\rangle t_{k}(x), \quad n \geq 3,
$$

where $\left\langle f, t_{k}\right\rangle=\frac{1}{2 \pi} \int_{0}^{2 \pi} f(x) t_{k}(x) \mathrm{d} x$ are Fourier coefficients of the function $f$ with respect to the basis $\left\{t_{k}\right\}_{k=0}^{\infty}$. It is convenient for us to put $\sigma_{n}(f, x) \equiv 0$ if $n=0,1,2$.

We also use the following representation of the operators $\sigma_{n}$ :

$$
\sigma_{n}(f, x)=\frac{1}{2 \pi} \int_{0}^{2 \pi} f(y) K_{n}(x, y) \mathrm{d} y
$$

where $K_{n}(x, y)=\sum_{k=0}^{2 N_{n-2}-1} t_{k}(x) t_{k}(y)$.

Using a similar technique as in Prestin and Selig [7], one can prove that the system of polynomials $\left\{t_{k}\right\}_{k=0}^{\infty}$ is an orthogonal trigonometric Schauder basis in the space $L_{2 \pi}^{\infty}$. In view of Theorem 9 [4, p. 12] and Theorem $6\left[4\right.$, p. 10] we get that $\left\{t_{k}\right\}_{k=0}^{\infty}$ is a Schauder basis in the space $L_{2 \pi}^{p}, 1 \leq p<\infty$, and

$$
\left\|\sigma_{n}(f, \cdot)\right\|_{p} \leq C\|f(\cdot)\|_{p}
$$

with some constant $C>0$.

By $\mathbb{T}_{n}, n \in \mathbb{N}$, we denote the set of all trigonometric polynomials of the form

$$
T(x)=\sum_{k=0}^{n}\left(a_{k} \cos k x+b_{k} \sin k x\right), x \in \mathbb{R} .
$$

From (2.3) and the representation of the kernel $K_{n}$ [7, p. 421], it can be derived that

$$
\sigma_{n}(T, \cdot)=T(\cdot), \quad T \in \mathbb{T}_{2^{n-1}}
$$

Let

$$
E_{n}(f)_{p}:=\inf _{T \in \mathbb{T}_{n}}\|f-T\|_{p}
$$

be the best approximation of a function $f \in L_{2 \pi}^{p}$ by trigonometric polynomials from $\mathbb{T}_{n}$.

A sequence of linear operators $U_{n}: L_{2 \pi}^{p} \rightarrow \mathbb{T}_{2^{n}}, n \in \mathbb{N}_{0}$, is called a sequence of near best approximation (with the constant $\lambda>0$ ) for $L_{2 \pi}^{p}$ if it satisfies the following condition:

$$
\left\|f-U_{n}(f)\right\|_{p} \leq \lambda E_{2^{n-1}}(f)_{p} .
$$


For example, in the case $p=\infty$ the operators of de la Vallée Poussin $V_{n}^{2 n}$ determine a sequence of near best approximation with the constant $\lambda=\frac{4}{3}+\frac{2 \sqrt{3}}{\pi}$ [12, Chap. 5, §2].

The following lemma results from the properties (2.4) and (2.5).

Lemma 2.1. $\left\{\sigma_{n}(f)\right\}_{n=0}^{\infty}$ is a sequence of near best approximation (with some constant) for $L_{2 \pi}^{p}, 1 \leq p \leq \infty$.

Further, for $f \in L_{2 \pi}^{p}, 1 \leq p \leq \infty$, we define operators $\tau_{n}$, $n \in \mathbb{N}_{0}$, as follows:

$$
\tau_{n}(f, x)=\sigma_{n+3}(f, x)-\sigma_{n+2}(f, x), x \in \mathbb{R}
$$

From Lemma 2.1 it is easy to see that a function $f \in L_{2 \pi}^{p}$, $1 \leq p \leq \infty$, can be represented by the series

$$
f(x)=\sum_{n=0}^{\infty} \tau_{n}(f, x), x \in \mathbb{R}
$$

where convergence is understood in the metric of the space $L_{2 \pi}^{p}$.

Using the definition of the operators $\tau_{n}$, we can represent them in the following form:

$$
\tau_{0}(f, x)=\sum_{k=0}^{2 N_{1}-1}\left\langle f, t_{k}\right\rangle t_{k}(x)=\sum_{s=0}^{2 N_{0}-1}\left\langle f, \psi_{0, s}\right\rangle \psi_{0, s}(x)
$$

and for $n \in \mathbb{N}$

$$
\tau_{n}(f, x)=\sum_{2 N_{n} \leq k<2 N_{n+1}-1}\left\langle f, t_{k}\right\rangle t_{k}(x)=\sum_{s=0}^{2 N_{n}-1}\left\langle f, \psi_{n, s}\right\rangle \psi_{n, s}(x) .
$$

From (2.6)-(2.8) we get representation (1.4).

\section{FORMULATIONS OF THE MAIN RESULTS}

In this section we formulate the main results of this paper. Let us first explain the relationship between the local Besov spaces $B_{p, \theta}^{\alpha}\left(x_{0}\right)$ and the behavior of the operators $\tau_{n}$ near the point $x_{0}$. This behavior will be described by the condition that certain norms of the operators belong to a sequential version of the Besov spaces.

Theorem 3.1. Let $1 \leq p \leq \infty, f \in L_{2 \pi}^{p}, x_{0} \in[0,2 \pi), 0<\alpha<1$, $0<\theta \leq \infty$. The following statements are equivalent:

(a) $f \in B_{p, \theta}^{\alpha}\left(x_{0}\right)$;

(b) There exists an interval $I \subset \mathbb{R},|I|<2 \pi$, centered at $x_{0}$ such that the sequence $\left\{\left\|\tau_{n}(f, \cdot)\right\|_{I, p}\right\}_{n=0}^{\infty} \in b_{\theta, \alpha}$;

(c) There exists an interval $I \subset \mathbb{R},|I|<2 \pi$, centered at $x_{0}$ such that for every infinitely differentiable on $\mathbb{R}$ function $\zeta$ supported on $I$ and extended as a $2 \pi$-periodic function, the sequence $\left\{\left\|\tau_{n}(f \zeta, \cdot)\right\|_{p}\right\}_{n=0}^{\infty} \in b_{\theta, \alpha}$.
Let further for $1 \leq p \leq \infty$ and a segment $I,\left\{c_{n}(I, p)\right\}_{n=0}^{\infty}$ be a sequence defined by the formula (1.5). And let $\zeta$ be some infinitely differentiable on $\mathbb{R}$ function supported on $I$ and extended as a $2 \pi$-periodic function. By $\left\{d_{n}(I, \zeta, p)\right\}_{n=0}^{\infty}$ we denote the sequence:

$$
d_{n}(I, \zeta, p):= \begin{cases}N_{n}^{1 / 2-1 / p}\left(\sum_{s=0}^{2 N_{n}-1}\left|a_{n, s}(f \zeta)\right|^{p}\right)^{1 / p}, & 1 \leq p<\infty, \\ N_{n}^{1 / 2} \max _{s=0, \ldots, 2 N_{n}-1}\left|a_{n, s}(f \zeta)\right|, & p=\infty .\end{cases}
$$

Theorem 3.2. Let $1 \leq p \leq \infty, f \in L_{2 \pi}^{p}, x_{0} \in[0,2 \pi), 0<\alpha<1$, $0<\theta \leq \infty$. The following statements are equivalent:

(a) $f \in B_{p, \theta}^{\alpha}\left(x_{0}\right)$;

(b) There exists an interval $I \subset \mathbb{R},|I|<2 \pi$, centered at $x_{0}$ such that the sequence $\left\{c_{n}(I, p)\right\}_{n=0}^{\infty} \in b_{\theta, \alpha}$;

(c) There exists an interval $I \subset \mathbb{R},|I|<2 \pi$, centered at $x_{0}$ such that for every infinitely differentiable on $\mathbb{R}$ function $\zeta$ supported on $I$ and extended as a $2 \pi$-periodic function, the sequence $\left\{d_{n}(I, \zeta, p)\right\}_{n=0}^{\infty} \in b_{\theta, \alpha}$.

This theorem is the discrete version of Theorem 3.1 in the sense that the $L_{2 \pi}^{p}$ norm of the operators $\tau_{n}$ is replaced by a corresponding discrete norm (see Theorem 4.1).

For classical Besov spaces $B_{p, \theta}^{\alpha *}, \alpha>0$, we can obtain a result similar to Theorems 3.1 and 3.2 which is essentially of the same kind as (1.1) and (1.2). To formulate this equivalence we introduce the following sequence

$$
a_{n}(p):= \begin{cases}N_{n}^{1 / 2-1 / p}\left(\sum_{s=0}^{2 N_{n}-1}\left|a_{n, s}(f)\right|^{p}\right)^{1 / p}, & 1 \leq p<\infty, \\ N_{n}^{1 / 2} \max _{s=0, \ldots, 2 N_{n}-1}\left|a_{n, s}(f)\right|, & p=\infty .\end{cases}
$$

Theorem 3.3. Let $1 \leq p \leq \infty, f \in L_{2 \pi}^{p}, \alpha>0,0<\theta \leq \infty$. The following statements are equivalent:
(a) $f \in B_{p, \theta}^{\alpha *}$;
(b) The sequence $\left\{\left\|\tau_{n}(f, \cdot)\right\|_{p}\right\}_{n=0}^{\infty} \in b_{\theta, \alpha}$;
(c) The sequence $\left\{a_{n}(p)\right\}_{n=0}^{\infty} \in b_{\theta, \alpha}$.

\section{THE MAIN AUXILIARY STATEMENTS}

\subsection{A Property of the Kernel $K_{n}(x, y)$}

In this subsection we present some estimates for the kernel $K_{n}(x, y)$. Further, by $\mathbf{V}[f]$ we mean the total variation of a function $f: \mathbb{R} \rightarrow \mathbb{R}$ and by $\mathbf{V}_{a}^{b}[f]$ the total variation of $f$ defined on the segment $[a, b]$.

Lemma 4.1. There exists a constant $C>0$ such that for every $n \in \mathbb{N}, n \geq 3$ :

$$
\left|K_{n}(x, y)\right| \leq \frac{C}{2^{n}|x-y|^{2}}, \quad 0<|x-y| \leq 2 \pi .
$$


Proof. Let $N=3 \cdot 2^{n-2}, M=2^{n-2}, n \geq 3$. From Selig [9, pp. 91-93] it is known that

$$
K_{n}(x, y)=Q_{N}^{M}(y-x)+\cos N(y+x) R_{M}(y-x)
$$

where

$$
\begin{aligned}
Q_{N}^{M}(x)= & 1+2 \sum_{k=1}^{N-M} \cos k x+\sum_{k=-M+1}^{M-1} \frac{(M-k)^{2}}{M^{2}+k^{2}} \cos (N+k) x \\
= & \sum_{k=-N+M}^{N-M} \cos k x \\
& +\frac{1}{2} \sum_{|k|=N-M+1}^{N+M-1} \frac{(M-(|k|-N))^{2}}{M^{2}+(|k|-N)^{2}} \cos k x, \\
R_{M}(x)= & 1+2 \sum_{k=1}^{M-1} \frac{M^{2}-k^{2}}{M^{2}+k^{2}} \cos k x \\
= & \sum_{k=-M+1}^{M-1} \frac{M^{2}-k^{2}}{M^{2}+k^{2}} \cos k x .
\end{aligned}
$$

Defining continuous coefficient functions by

$$
\begin{gathered}
g(x)= \begin{cases}\frac{1-x^{2}}{1+x^{2}}, & -1 \leq x \leq 1, \\
0, & \text { else }\end{cases} \\
f_{\beta}(x)= \begin{cases}1, & |x| \leq 1-\beta, \\
\frac{(\beta-(|x|-1))^{2}}{2\left(\beta^{2}+(|x|-1)^{2}\right)}, & 1-\beta \leq|x| \leq 1+\beta, \\
0, & \text { else, }\end{cases}
\end{gathered}
$$

where $\beta \in(0,1)$, we can rewrite the polynomials $R_{M}$ and $Q_{N}^{M}$ in the following forms:

$$
R_{M}(x)=\sum_{k \in \mathbb{Z}} g\left(\frac{k}{M}\right) \cos k x, Q_{N}^{M}(x)=\sum_{k \in \mathbb{Z}} f_{\frac{M}{N}}\left(\frac{k}{N}\right) \cos k x .
$$

Let us first estimate $\left|R_{M}(y-x)\right|$. Using Proposition 2.2 [13], for $0<|x-y| \leq 2 \pi$ we get

$$
\begin{aligned}
\left|R_{M}(y-x)\right|= & \left|\sum_{k \in \mathbb{Z}} g\left(\frac{k}{M}\right) \cos k(y-x)\right| \\
& \leq \frac{C}{|x-y|^{2}} \sum_{k \in \mathbb{Z}}\left|\Delta^{2} g\left(\frac{k}{M}\right)\right|,
\end{aligned}
$$

where $\Delta^{2} g\left(\frac{k}{M}\right)=g\left(\frac{k}{M}\right)-2 g\left(\frac{k+1}{M}\right)+g\left(\frac{k+2}{M}\right)$.

Then, using the mean value theorem, we obtain that there exist points $l_{k} \in(k, k+1), k \in \mathbb{Z}$, such that

$$
\begin{aligned}
\Delta^{2} g\left(\frac{k}{M}\right) & =g\left(\frac{k}{M}\right)-2 g\left(\frac{k+1}{M}\right)+g\left(\frac{k+2}{M}\right) \\
& =\frac{1}{M}\left(g^{\prime}\left(\frac{l_{k+1}}{M}\right)-g^{\prime}\left(\frac{l_{k}}{M}\right)\right) .
\end{aligned}
$$

Since the system of the points $\left\{\frac{l_{k}}{M}, k \in \mathbb{Z}\right\}$ is some partition of the real line and $g$ is a function having a first derivative of finite total variation $\mathbf{V}\left[g^{\prime}\right]$, it holds that

$$
\begin{aligned}
\sum_{k \in \mathbb{Z}}\left|\Delta^{2} g\left(\frac{k}{M}\right)\right|= & \sum_{k \in \mathbb{Z}} \frac{1}{M}\left|\left(g^{\prime}\left(\frac{l_{k+1}}{M}\right)-g^{\prime}\left(\frac{l_{k}}{M}\right)\right)\right| \\
& \leq \frac{1}{M} \mathbf{V}\left[g^{\prime}\right]=\frac{C}{2^{n}} \mathbf{V}\left[g^{\prime}\right] .
\end{aligned}
$$

Since $g^{\prime}=0$ outside of the segment $[-1,1]$, it holds that $\mathbf{V}\left[g^{\prime}\right]=$ $\mathbf{V}_{-1}^{1}\left[g^{\prime}\right]$.

From the first and second derivatives

$$
g^{\prime}(x)=-\frac{4 x}{\left(1+x^{2}\right)^{2}}, \quad g^{\prime \prime}(x)=\frac{4\left(3 x^{2}-1\right)}{\left(1+x^{2}\right)^{3}},
$$

we see that $g^{\prime \prime}=0$ at the points $-\frac{\sqrt{3}}{3}$ and $\frac{\sqrt{3}}{3}$ and $g^{\prime}$ is monotonously increasing on $\left[-1,-\frac{\sqrt{3}}{3}\right]$ and on $\left[\frac{\sqrt{3}}{3}, 1\right]$ and monotonously decreasing on $\left[-\frac{\sqrt{3}}{3}, \frac{\sqrt{3}}{3}\right]$. Therefore, the extrema are at $-\frac{\sqrt{3}}{3}$ and $\frac{\sqrt{3}}{3}$ :

$$
\sup _{x \in[-1,1]} g^{\prime}(x)=g^{\prime}\left(-\frac{\sqrt{3}}{3}\right)=-g^{\prime}\left(\frac{\sqrt{3}}{3}\right)=\frac{3 \sqrt{3}}{4} .
$$

Since $g^{\prime}(-1)>0$ and $g^{\prime}(1)<0$, the total variation of $g^{\prime}$ is

$$
\mathbf{V}\left[g^{\prime}\right]=\mathbf{V}_{-1}^{1}\left[g^{\prime}\right]=4 \sup _{x \in[-1,1]} g^{\prime}(x)=3 \sqrt{3}
$$

From (4.2), (4.3) and (4.4), we get

$$
\left|R_{M}(y-x)\right| \leq \frac{C}{2^{n}|x-y|^{2}}, \quad 0<|x-y| \leq 2 \pi .
$$

Analogously, we can estimate $\left|Q_{N}^{M}(y-x)\right|$. Let us calculate $\mathbf{V}\left[f_{\beta}^{\prime}\right]$ where

$$
f_{\beta}^{\prime}(x)= \begin{cases}-\frac{\beta\left(\beta^{2}-(x-1)^{2}\right)}{\beta^{2}+(x-1)^{2}}, & 1-\beta \leq x \leq 1+\beta \\ \frac{\beta\left(\beta^{2}-(-x-1)^{2}\right)}{\beta^{2}+(-x-1)^{2}}, & -1-\beta \leq x \leq-1+\beta \\ 0, & \text { else. }\end{cases}
$$

Since $f_{\beta}^{\prime}=0$ outside of the segments $[-1-\beta,-1+\beta]$ and $[1-\beta, 1+\beta]$ and $f_{\beta}^{\prime}$ is an odd function, it holds that $\mathbf{V}\left[f_{\beta}^{\prime}\right]=$ $2 \mathbf{V}_{-1-\beta}^{-1+\beta}\left[f_{\beta}^{\prime}\right]$.

From the second derivative

$f_{\beta}^{\prime \prime}(x)=\frac{2 \beta(1+x)\left(-3 \beta^{2}+(1+x)^{2}\right)}{\left(\beta^{2}+(1+x)^{2}\right)^{3}}, x \in[-1-\beta,-1+\beta]$,

we can see that $f_{\beta}^{\prime \prime}=0$ at the point -1 and $f_{\beta}^{\prime}$ is monotonously increasing on $[-1-\beta,-1]$ and monotonously decreasing on $[-1,-1+\beta]$. This means that

$$
\sup _{x \in[-1-\beta,-1+\beta]} f_{\beta}^{\prime}(x)=f_{\beta}^{\prime}(-1)=\frac{1}{\beta} .
$$


Since $f_{\beta}^{\prime}(-1-\beta)=f_{\beta}^{\prime}(-1+\beta)=0$, it holds that

$$
\mathbf{V}_{-1-\beta}^{-1+\beta}\left[f_{\beta}^{\prime}\right]=2 \sup _{x \in[-1-\beta,-1+\beta]} f_{\beta}^{\prime}(x)=\frac{2}{\beta},
$$

and the total variation of $f_{\beta}^{\prime}$ is

$$
\mathbf{V}\left[f_{\beta}^{\prime}\right]=\frac{4}{\beta} .
$$

Then, using Proposition 2.2 [13], for $0<|x-y| \leq 2 \pi$ we get

$$
\begin{aligned}
\left|Q_{N}^{M}(y-x)\right|= & \left|\sum_{k \in \mathbb{Z}} f_{\frac{M}{N}}\left(\frac{k}{N}\right) \cos k(y-x)\right| \\
& \leq \frac{C}{|x-y|^{2}} \sum_{k \in \mathbb{Z}}\left|\Delta^{2} f_{\frac{M}{N}}\left(\frac{k}{N}\right)\right|,
\end{aligned}
$$

where $\Delta^{2} f_{\frac{M}{N}}\left(\frac{k}{N}\right)=f_{\frac{M}{N}}\left(\frac{k}{N}\right)-2 f_{\frac{M}{N}}\left(\frac{k+1}{N}\right)+f_{\frac{M}{N}}\left(\frac{k+2}{N}\right)$.

From the mean value theorem it follows that there exist points $s_{k} \in(k, k+1), k \in \mathbb{Z}$, such that

$$
\Delta^{2} f_{\frac{M}{N}}\left(\frac{k}{N}\right)=\frac{1}{N}\left(f_{\frac{M}{N}}^{\prime}\left(\frac{s_{k+1}}{N}\right)-f_{\frac{M}{N}}^{\prime}\left(\frac{s_{k}}{N}\right)\right) .
$$

Since the system of the points $\left\{\frac{s_{k}}{N}, k \in \mathbb{Z}\right\}$ is some partition of the real line and $f_{\frac{M}{N}}$ is a function having a first derivative of finite total variation, from (4.6) and (4.8) it can be derived that

$$
\begin{aligned}
\sum_{k \in \mathbb{Z}}\left|\Delta^{2} f_{\frac{M}{N}}\left(\frac{k}{N}\right)\right| & \leq \sum_{k \in \mathbb{Z}} \frac{1}{N}\left|\left(f_{\frac{M}{N}}^{\prime}\left(\frac{s_{k+1}}{N}\right)-f_{\frac{M}{N}}^{\prime}\left(\frac{s_{k}}{N}\right)\right)\right| \\
& \leq \frac{1}{N} \mathbf{V}\left[f_{\frac{M}{N}}^{\prime}\right]=\frac{C}{2^{n}} .
\end{aligned}
$$

In view of (4.7) and (4.9), we have

$$
\left|Q_{N}^{M}(y-x)\right| \leq \frac{C}{2^{n}|x-y|^{2}}, \quad 0<|x-y| \leq 2 \pi .
$$

Finally, from (4.1), (4.5) and (4.10) we conclude that

$$
\left|K_{n}(x, y)\right| \leq \frac{C}{2^{n}|x-y|^{2}}, \quad 0<|x-y| \leq 2 \pi .
$$

\subsection{A Property of the Spaces $V_{n}$ and $W_{n}$}

In this subsection we formulate and prove the main auxiliary statement. Let us make some preparations for this. We use the Minkowski inequality in the following form:

$$
\left(\sum_{l=1}^{m}\left|\int_{a}^{b} f_{l}(x) \mathrm{d} x\right|^{p}\right)^{1 / p} \leq \int_{a}^{b}\left(\sum_{l=1}^{m}\left|f_{l}(x)\right|^{p}\right)^{1 / p} \mathrm{~d} x, 1 \leq p<\infty
$$

(under appropriate conditions on the functions $f_{l}$ which appear above). One can prove this inequality by using a similar technique as in the proof of the generalized Minkowski inequality [14, pp. 18-19].

Since the coefficient functions $g_{1}$ and $g_{2}$ defined by the formulas (2.1) and (2.2) on the segments of length $2 \pi$ have the first derivatives (on corresponding segments) of finite total variation, by similar techniques as in Lemma 4.3 [7] one can prove the following estimates (with some constants $C_{1}$ and $C_{2}$ ):

$$
\begin{aligned}
& \left\|\sum_{s=0}^{2 N_{n}-1}\left|\varphi_{n, s}(\cdot)\right|\right\|_{\infty} \leq C_{1} \sqrt{N_{n}}, \\
& \left\|\sum_{s=0}^{2 N_{n}-1}\left|\psi_{n, s}(\cdot)\right|\right\|_{\infty} \leq C_{2} \sqrt{N_{n}} .
\end{aligned}
$$

Theorem 4.1. Let $n \in \mathbb{N}$ and $\left(a_{n, s}\right)_{s=0}^{2 N_{n}-1}$ be an arbitrary sequence of real numbers. Then, there exist constants $C_{i}>0, i=1,2,3,4$, such that for $1 \leq p<\infty$ the following inequalities hold:

$$
\begin{aligned}
C_{1} 2^{n\left(\frac{1}{2}-\frac{1}{p}\right)}\left(\sum_{s=0}^{2 N_{n}-1}\left|a_{n, s}\right|^{p}\right)^{\frac{1}{p}} & \leq\left\|\sum_{s=0}^{2 N_{n}-1} a_{n, s} \psi_{n, s}(\cdot)\right\|_{p} \\
& \leq C_{2} 2^{n\left(\frac{1}{2}-\frac{1}{p}\right)}\left(\sum_{s=0}^{2 N_{n}-1}\left|a_{n, s}\right|^{p}\right)^{\frac{1}{p}} .
\end{aligned}
$$

For $p=\infty$ we have

$$
\begin{aligned}
C_{3} 2^{\frac{n}{2}} \max _{s=0, \ldots, 2 N_{n}-1}\left|a_{n, s}\right| & \leq\left\|\sum_{s=0}^{2 N_{n}-1} a_{n, s} \psi_{n, s}(\cdot)\right\|_{\infty} \\
& \leq C_{4} 2^{\frac{n}{2}} \max _{s=0, \ldots, 2 N_{n}-1}\left|a_{n, s}\right| .
\end{aligned}
$$

Proof. For the sake of simplicity we denote

$$
\tau(x)=\sum_{s=0}^{2 N_{n}-1} a_{n, s} \psi_{n, s}(x) .
$$

First, we prove the right-hand side of (4.14). For $p=1$ we have

$$
\begin{aligned}
& \|\tau(\cdot)\|_{1}=\frac{1}{2 \pi} \int_{0}^{2 \pi}\left|\sum_{s=0}^{2 N_{n}-1} a_{n, s} \psi_{n, s}(x)\right| \mathrm{d} x \\
& \leq \sum_{s=0}^{2 N_{n}-1}\left|a_{n, s}\right| \frac{1}{2 \pi} \int_{0}^{2 \pi}\left|\psi_{n, s}(x)\right| \mathrm{d} x \\
& =\sum_{s=0}^{2 N_{n}-1}\left|a_{n, s}\right|\left\|\psi_{n}\left(\cdot-\frac{s \pi}{N_{n}}\right)\right\|_{1}=\left\|\psi_{n}(\cdot)\right\|_{1} \sum_{s=0}^{2 N_{n}-1}\left|a_{n, s}\right|
\end{aligned}
$$

Let us estimate $\left\|\psi_{n}(\cdot)\right\|_{1}$. For the $L_{2 \pi}^{1}$-norm of a polynomial $T \in \mathbb{T}_{n}$ it holds that $[15$, p. 228]

$$
\|T(\cdot)\|_{1} \leq \sup _{x} \frac{2 \pi}{m} \sum_{s=0}^{m-1}\left|T\left(x-\frac{2 \pi s}{m}\right)\right|, \quad m \in \mathbb{N} .
$$


Applying this estimate to $\psi_{n}$ with $m=2 N_{n}$ and using the inequality (4.13), we obtain

$\left\|\psi_{n}(\cdot)\right\|_{1} \leq \frac{1}{2 N_{n}}\left\|\sum_{s=0}^{2 N_{n}-1}\left|\psi_{n}\left(\cdot-\frac{s \pi}{N_{n}}\right)\right|\right\|_{\infty} \leq \frac{C}{\sqrt{2 N_{n}}} \leq C 2^{-n / 2}$.

From (4.17) and (4.18) we derive

$$
\|\tau(\cdot)\|_{1} \leq C 2^{-n / 2} \sum_{s=0}^{2 N_{n}-1}\left|a_{n, s}\right| .
$$

Let $1<p<\infty$. Using the inequality of Hölder for sums

$$
\sum_{k}\left|a_{k} b_{k}\right| \leq\left(\sum_{k}\left|a_{k}\right|^{p}\right)^{1 / p}\left(\sum_{k}\left|b_{k}\right|^{p^{\prime}}\right)^{1 / p^{\prime}}, \frac{1}{p}+\frac{1}{p^{\prime}}=1,
$$

and the inequality (4.13), we obtain

$$
\begin{aligned}
\left|\sum_{s=0}^{2 N_{n}-1} a_{n, s} \psi_{n, s}(x)\right| & \leq \sum_{s=0}^{2 N_{n}-1}\left|a_{n, s}\right|\left|\psi_{n, s}(x)\right| \\
& =\sum_{s=0}^{2 N_{n}-1}\left|a_{n, s}\right|\left|\psi_{n, s}(x)\right|^{\frac{1}{p}+\frac{1}{p^{\prime}}} \\
& \leq\left(\sum_{s=0}^{2 N_{n}-1}\left|a_{n, s}\right|^{p}\left|\psi_{n, s}(x)\right|\right)^{\frac{1}{p}} \\
& \times\left(\sum_{s=0}^{2 N_{n}-1}\left|\psi_{n, s}(x)\right|\right)^{\frac{1}{p^{\prime}}} \\
& \leq\left(\sum_{s=0}^{2 N_{n}-1}\left|a_{n, s}\right|^{p}\left|\psi_{n, s}(x)\right|\right)^{\frac{1}{p}} \\
& \times\left(\sup _{x \in[0,2 \pi)}^{2 N_{n}-1}\left|\sum_{s=0}^{2 N_{n}-1}\right| \psi_{n, s}(x) \mid\right)^{\frac{1}{p^{\prime}}} \\
& \leq C 2^{\frac{n}{2 p^{\prime}}}\left(\sum_{s=0}^{\left.\left.2 a_{n, s}\right|^{p}\left|\psi_{n, s}(x)\right|\right)^{\frac{1}{p}} .}\right.
\end{aligned}
$$

Therefore,

$$
\left|\sum_{s=0}^{2 N_{n}-1} a_{n, s} \psi_{n, s}(x)\right|^{p} \leq C 2^{\frac{n p}{2 p^{\prime}}} \sum_{s=0}^{2 N_{n}-1}\left|a_{n, s}\right|^{p}\left|\psi_{n, s}(x)\right| .
$$

In view of (4.18), we have

$$
\begin{aligned}
\|\tau(\cdot)\|_{p}^{p} & =\frac{1}{2 \pi} \int_{0}^{2 \pi}\left|\sum_{s=0}^{2 N_{n}-1} a_{n, s} \psi_{n, s}(x)\right|^{p} \mathrm{~d} x \\
& \leq C 2^{\frac{n p}{2 p^{\prime}}} \sum_{s=0}^{2 N_{n}-1}\left|a_{n, s}\right|^{p} \frac{1}{2 \pi} \int_{0}^{2 \pi}\left|\psi_{n, s}(x)\right| \mathrm{d} x \\
& =C 2^{\frac{n p}{2 p^{\prime}}}\left\|\psi_{n}(\cdot)\right\|_{1} \sum_{s=0}^{2 N_{n}-1}\left|a_{n, s}\right|^{p} \\
& \leq C 2^{\frac{n p}{2 p^{\prime}}} 2^{-\frac{n}{2}} \sum_{s=0}^{2 N_{n}-1}\left|a_{n, s}\right|^{p}=C 2^{n p\left(\frac{1}{2}-\frac{1}{p}\right)} \sum_{s=0}^{2 N_{n}-1}\left|a_{n, s}\right|^{p},
\end{aligned}
$$

and this implies that

$$
\|\tau(\cdot)\|_{p} \leq C 2^{n\left(\frac{1}{2}-\frac{1}{p}\right)}\left(\sum_{s=0}^{2 N_{n}-1}\left|a_{n, s}\right|^{p}\right)^{1 / p}
$$

Now, we prove the left-hand side of (4.14). Taking the inner product in (4.16) with $T_{n}^{s} \psi_{n}$, we get that for all $s=0, \ldots, 2 N_{n}-1$

$$
a_{n, s}=\left\langle\tau, \psi_{n, s}\right\rangle=\frac{1}{2 \pi} \int_{0}^{2 \pi} \tau(x) \psi_{n}\left(x-\frac{s \pi}{N_{n}}\right) \mathrm{d} x
$$

Let $p=1$. Using (4.19) and the estimation (4.13), we obtain

$$
\begin{aligned}
\sum_{s=0}^{2 N_{n}-1}\left|a_{n, s}\right| & =\sum_{s=0}^{2 N_{n}-1} \frac{1}{2 \pi}\left|\int_{0}^{2 \pi} \tau(x) \psi_{n}\left(x-\frac{s \pi}{N_{n}}\right) \mathrm{d} x\right| \\
& \leq \frac{1}{2 \pi} \int_{0}^{2 \pi}|\tau(x)| \sum_{s=0}^{2 N_{n}-1}\left|\psi_{n}\left(x-\frac{s \pi}{N_{n}}\right)\right| \mathrm{d} x \\
& \leq \sup _{x \in[0,2 \pi)} \sum_{s=0}^{2 N_{n}-1}\left|\psi_{n}\left(x-\frac{s \pi}{N_{n}}\right)\right| \\
& \times \frac{1}{2 \pi} \int_{0}^{2 \pi}|\tau(x)| \mathrm{d} x \leq C 2^{n / 2}\|\tau(\cdot)\|_{1} .
\end{aligned}
$$

Finally, $C 2^{-n / 2} \sum_{s=0}^{2 N_{n}-1}\left|a_{n, s}\right| \leq\|\tau(\cdot)\|_{1}$.

Let now $1<p<\infty$. From (4.19) and the inequality (4.11) we derive that

$$
\begin{aligned}
\left(\sum_{s=0}^{2 N_{n}-1}\left|a_{n, s}\right|^{p}\right)^{1 / p} & =\left(\sum_{s=0}^{2 N_{n}-1}\left|\frac{1}{2 \pi} \int_{0}^{2 \pi} \tau(x) \psi_{n}\left(x-\frac{s \pi}{N_{n}}\right) \mathrm{d} x\right|^{p}\right)^{1 / p} \\
& =\left(\sum_{s=0}^{2 N_{n}-1}\left|\frac{1}{2 \pi} \int_{0}^{2 \pi} \tau\left(x-\frac{s \pi}{N_{n}}\right) \psi_{n}(x) \mathrm{d} x\right|^{p}\right)^{1 / p} \\
& \leq \frac{1}{2 \pi} \int_{0}^{2 \pi}\left(\sum_{s=0}^{2 N_{n}-1}\left|\tau\left(x-\frac{s \pi}{N_{n}}\right)\right|^{p}\left|\psi_{n}(x)\right|^{p}\right)^{1 / p} \mathrm{~d} x \\
& =\frac{1}{2 \pi} \int_{0}^{2 \pi}\left|\psi_{n}(x)\right|\left(\sum_{s=0}^{2 N_{n}-1}\left|\tau\left(x-\frac{s \pi}{N_{n}}\right)\right|^{p}\right)^{1 / p} \mathrm{~d} x \\
& \leq \sup _{x \in[0,2 \pi)}\left(\sum_{s=0}^{2 N_{n}-1}\left|\tau\left(x-\frac{s \pi}{N_{n}}\right)\right|^{p}\right)^{1 / p}\left\|\psi_{n}(\cdot)\right\|_{1} \\
& \leq C 2^{\frac{n}{p}} \sup _{x \in[0,2 \pi)}\left(\frac{1}{2 N_{n}} \sum_{s=0}^{2 N_{n}-1}\left|\tau\left(x-\frac{s \pi}{N_{n}}\right)\right|^{p}\right)^{1 / p}\left\|\psi_{n}(\cdot)\right\|_{1} .
\end{aligned}
$$

For the $L_{2 \pi}^{p}$-norm of a polynomial $T \in \mathbb{T}_{n}$ we use the following inequality [15, p. 228]:

$\sup _{x}\left(\frac{2 \pi}{m} \sum_{s=0}^{m-1}\left|T\left(x-\frac{2 \pi s}{m}\right)\right|^{p}\right)^{\frac{1}{p}} \leq\left(1+\frac{2 n \pi}{m}\right)\|T(\cdot)\|_{p}, m \in \mathbb{N}$. 
Applying this estimation to $\tau$ with $m=2 N_{n}$ and using the inequality (4.18), we obtain that

$$
\left(\sum_{s=0}^{2 N_{n}-1}\left|a_{n, s}\right|^{p}\right)^{1 / p} \leq C 2^{n\left(\frac{1}{p}-\frac{1}{2}\right)}\|\tau(\cdot)\|_{p}
$$

Let $p=\infty$. The right-hand side of the inequality (4.15) follows from obvious inequalities and the inequality (4.13):

$$
\begin{aligned}
& \|\tau(\cdot)\|_{\infty}=\sup _{x \in[0,2 \pi)}\left|\sum_{s=0}^{2 N_{n}-1} a_{n, s} \psi_{n, s}(x)\right| \\
& \leq \sup _{x \in[0,2 \pi)} \sum_{s=0}^{2 N_{n}-1}\left|a_{n, s}\right|\left|\psi_{n, s}(x)\right| \\
& \leq \max _{s=0, \ldots, 2 N_{n}-1}\left|a_{n, s}\right| \sup _{x \in[0,2 \pi]} \sum_{s=0}^{2 N_{n}-1}\left|\psi_{n}\left(x-\frac{s \pi}{N_{n}}\right)\right| \\
& =\max _{s=0, \ldots, 2 N_{n}-1}\left|a_{n, s}\right|\left|\sum_{s=0}^{2 N_{n}-1}\right| \psi_{n}\left(\cdot-\frac{s \pi}{N_{n}}\right) \mid \|_{\infty} \\
& \leq C \sqrt{2 N_{n}} \max _{s=0, \ldots, 2 N_{n}-1}\left|a_{n, s}\right| \leq C 2^{n / 2} \max _{s=0, \ldots, 2 N_{n}-1}\left|a_{n, s}\right| .
\end{aligned}
$$

Let us prove the left-hand side of (4.15). From the inequality (4.19) for $s=0, \ldots, 2 N_{n}-1$ we get

$$
\begin{aligned}
\left|a_{n, s}\right| & \leq \frac{1}{2 \pi} \int_{0}^{2 \pi}|\tau(x)|\left|\psi_{n}\left(x-s \pi / N_{n}\right)\right| \mathrm{d} x \\
& \leq\|\tau\|_{\infty}\left\|\psi_{n}\right\|_{1} \leq C 2^{-n / 2}\|\tau\|_{\infty} .
\end{aligned}
$$

Finally, we conclude:

$$
C 2^{n / 2} \max _{s=0, \ldots, 2 N_{n}-1}\left|a_{n, s}\right| \leq\|\tau\|_{\infty} .
$$

We formulated and proved Theorem 4.1 for a polynomial $\tau \in W_{n}$, but using the same techniques and the inequality (4.12) instead of (4.13) in the corresponding places of the proof, one can prove a similar theorem for a polynomial $\tau \in V_{n}$.

\section{PROOF OF THE MAIN RESULTS}

\subsection{Proof of Theorem 3.1}

In order to prove Theorem 3.1, we need some known statements from the paper Mhaskar and Prestin [6], Theorem A and Lemma A, and the following Lemma 5.1. Note that in the proofs of this Lemma and Theorem 3.1 we use similar considerations as in the proofs of Lemma 4.2 and Theorem 2.1 in Mhaskar and Prestin [6].

Theorem A. Let $1 \leq p \leq \infty, f \in L_{2 \pi}^{p}, \alpha>0,0<$ $\theta \leq \infty$, and $\left\{U_{n}\right\}$ be a sequence of near best approximation (with some constant) for $L_{2 \pi}^{p}$. The following statements are equivalent: (a) $f \in B_{p, \theta}^{\alpha *}$;

(b) $\left\{E_{2^{n}}(f)_{p}\right\} \in b_{\theta, \alpha}$;

(c) $\left\{\left\|U_{n}(f)-U_{n-1}(f)\right\|_{p}\right\} \in b_{\theta, \alpha}$.

Lemma A. Let $1 \leq p \leq \infty, f \in L_{2 \pi}^{p}, \alpha>0,0<\theta \leq$ $\infty$, and $\left\{U_{n}\right\}$ be a sequence of near best approximation (with some constant) for $L_{2 \pi}^{p}$. If, for some interval I centered at $x_{0}$, the sequence $\left\{\left\|U_{n}(f)-U_{n-1}(f)\right\|_{I, p}\right\} \in b_{\theta, \alpha}$, then for every infinitely differentiable on $\mathbb{R}$ function $\zeta$ supported on $I$ and extended $2 \pi$ periodically, the function $f \zeta$ is in $B_{p, \theta}^{\alpha *}$.

Lemma 5.1. Let $I \subset \mathbb{R},|I|<2 \pi$, be an interval centered at $x_{0}$, $J_{1}$ and $J$ be intervals centered at $x_{0}$ such that $J \subset J_{1} \subset I, \zeta$ be an infinitely differentiable on $\mathbb{R}$ function supported on $I$ and extended as a $2 \pi$-periodic function such that $\zeta(x)=1$ for all $x \in J_{1}$ and let $f \in L_{2 \pi}^{1}$. Then, for the operator

$$
\sigma_{n}(f, x)=\frac{1}{2 \pi} \int_{0}^{2 \pi} f(y) K_{n}(x, y) \mathrm{d} y, \quad n \geq 3,
$$

we have

$$
\left\|\sigma_{n}((1-\zeta) f, \cdot)\right\|_{J, \infty} \leq \frac{C(I, \zeta, f)}{2^{n}} .
$$

Proof. Without loss of generality we can assume that $J_{1}$ is an interval with length equal to $|I| / 2$ and $J$ is an interval with length equal to $|I| / 4$. For $x \in J$, we have

$$
\begin{aligned}
\left|\sigma_{n}((1-\zeta) f, x)\right| & \leq \frac{1}{2 \pi} \int_{0}^{2 \pi}\left|f(y)(1-\zeta(y)) K_{n}(x, y)\right| \mathrm{d} y \\
& =\frac{1}{2 \pi} \int_{[0,2 \pi) \backslash J_{1}}|f(y)(1-\zeta(y))|\left|K_{n}(x, y)\right| \mathrm{d} y .
\end{aligned}
$$

Since $x \in J$ and $y \in[0,2 \pi) \backslash J_{1}$, then $|I| / 8<|x-y|$. Using Lemma 4.1, we get

$$
\begin{aligned}
\left|\sigma_{n}((1-\zeta) f, x)\right| & \leq \frac{1}{2 \pi} \int_{[0,2 \pi) \backslash J_{1}}|f(y)(1-\zeta(y))| \frac{C}{2^{n}|x-y|^{2}} \mathrm{~d} y \\
& \leq \frac{C}{2 \pi(|I| / 8)^{2}} \frac{1}{2^{n}} \int_{[0,2 \pi) \backslash J_{1}}|f(y)(1-\zeta(y))| \mathrm{d} y \\
& \leq \frac{C(I, \zeta, f)}{2^{n}} .
\end{aligned}
$$

Therefore,

$$
\left\|\sigma_{n}((1-\zeta) f, \cdot)\right\|_{J, \infty} \leq \frac{C(I, \zeta, f)}{2^{n}} .
$$

Proof of Theorem 3.1. Let part (a) hold. In view of Proposition A, it is equivalent to the fact that there exists an interval $I$ centered at $x_{0}$ such that for every infinitely differentiable on $\mathbb{R}$ function 
$\zeta$ supported on $I$ and extended as a $2 \pi$-periodic function, the function $f \zeta$ is in $B_{p, \theta}^{\alpha *}$. According to Theorem A (with $\sigma_{n}$ instead of $U_{n}$ ) it is equivalent to part (c). Thus, parts (a) and (c) are equivalent.

Let part (c) hold and let $I$ be the interval chosen as in that part, let $J_{1}$ and $J$ be intervals centered at $x_{0}$ such that $J \subset J_{1} \subset I$ and $\zeta$ be an infinitely differentiable on $\mathbb{R}$ function supported on $I$ and extended as $2 \pi$-periodic such that $\zeta(x)=1$ for all $x \in J_{1}$.

From the obvious inequalities, we get

$$
\begin{aligned}
\left\|\tau_{n}(f, \cdot)\right\|_{J, p} & \leq\left\|\tau_{n}(f \zeta, \cdot)\right\|_{J, p}+\left\|\tau_{n}(f(1-\zeta), \cdot)\right\|_{J, p} \\
& \leq\left\|\tau_{n}(f \zeta, \cdot)\right\|_{p}+\left\|\tau_{n}(f(1-\zeta), \cdot)\right\|_{J, \infty} \\
& \leq\left\|\tau_{n}(f \zeta, \cdot)\right\|_{p} \\
& +\left\|\sigma_{n}(f(1-\zeta), \cdot)\right\|_{J, \infty}+\left\|\sigma_{n-1}(f(1-\zeta), \cdot)\right\|_{J, \infty},
\end{aligned}
$$

and using Lemma 5.1, we obtain

$$
\left\|\tau_{n}(f, \cdot)\right\|_{J, p} \leq\left\|\tau_{n}(f \zeta, \cdot)\right\|_{p}+\frac{C(I, \zeta, f)}{2^{n}} .
$$

Since $\alpha \in(0,1),\left\{2^{-n}\right\}_{n=1}^{\infty} \in b_{\theta, \alpha}$ and from the condition of part (c) we know that $\left\{\left\|\tau_{n}(f \zeta, \cdot)\right\|_{p}\right\}_{n=0}^{\infty} \in$ $b_{\theta, \alpha}$. Therefore, $\left\{\left\|\tau_{n}(f, \cdot)\right\|_{J, p}\right\}_{n=0}^{\infty} \in b_{\theta, \alpha}$ and part (b) is proved.

Let part (b) hold, and $I$ be the interval chosen as in that part and let $\zeta$ be an infinitely differentiable on $\mathbb{R}$ function supported on $I$ and extended as a $2 \pi$-periodic function. In view of Lemma A (applied with $\sigma_{n}$ in place of $U_{n}$ ), we get that $f \phi \in B_{p, \theta}^{\alpha *}$. According to Theorem A this means that $\left\{\left\|\tau_{n}(f \zeta, \cdot)\right\|_{p}\right\}_{n=0}^{\infty} \in b_{\theta, \alpha}$. This proves part (c).

\subsection{Proof of Theorem 3.2}

Let us first formulate and prove some auxiliary statements.

Lemma 5.2. There exist constants $C_{1}, C_{2}>0$ such that for every $n \in \mathbb{N}$ and $0<x \leq 2 \pi$ :

$$
\begin{aligned}
& \left|\varphi_{n}(x)\right| \leq \frac{C_{1}}{2^{3 n / 2}|x|^{2}}, \\
& \left|\psi_{n}(x)\right| \leq \frac{C_{2}}{2^{3 n / 2}|x|^{2}} .
\end{aligned}
$$

Proof. First, we prove estimation (5.1). Note that we can define the scaling functions $\varphi_{n}$ using an aperiodic coefficient function

$$
\varphi_{n}(\cdot)=\frac{1}{2 \sqrt{N_{n}}} \sum_{k \in \mathbb{Z}} g_{3}\left(\frac{k}{N_{n}}\right) \cos k \cdot
$$

where

$$
g_{3}(x)= \begin{cases}\sqrt{2}, & |x| \leq 1-\frac{1}{3} \\ \frac{1-3(|x|-1)}{\sqrt{1+9(|x|-1)^{2}}}, & 1-\frac{1}{3} \leq|x| \leq 1+\frac{1}{3} \\ 0, & \text { else. }\end{cases}
$$

Let us calculate $\mathbf{V}\left[g_{3}^{\prime}\right]$. Since $g_{3}^{\prime}=0$ outside of the segment $[-4 / 3,4 / 3]$, it holds that $\mathbf{V}\left[g_{3}^{\prime}\right]=\mathbf{V}_{-\frac{4}{3}}^{\frac{4}{3}}\left[g_{3}^{\prime}\right]$. From the first and second derivatives of $g_{3}$

$$
g_{3}^{\prime}(x)= \begin{cases}\frac{6-9 x}{\left(9 x^{2}-18 x+10\right)^{3 / 2}}, & \frac{2}{3} \leq x \leq \frac{4}{3} \\ -\frac{6+9 x}{\left(9 x^{2}+18 x+10\right)^{3 / 2}}, & -\frac{4}{3} \leq x \leq-\frac{2}{3}, \\ 0, & \text { else }\end{cases}
$$

$$
g_{3}^{\prime \prime}(x)= \begin{cases}\frac{9\left(18 x^{2}-27 x+8\right)}{\left(9 x^{2}-18 x+10\right)^{5 / 2}}, & \frac{2}{3} \leq x \leq \frac{4}{3} \\ -\frac{9\left(18 x^{2}+27 x+8\right)}{\left(9 x^{2}+18 x+10\right)^{5 / 2}}, & -\frac{4}{3} \leq x \leq-\frac{2}{3} \\ 0, & \text { else, }\end{cases}
$$

we get that $g_{3}^{\prime \prime}=0$ at the points $-\frac{1}{12}(9+\sqrt{17})$ and $-\frac{1}{12}(9+\sqrt{17})$ and $g_{3}^{\prime}$ is monotonously increasing on the segments $\left[-\frac{4}{3},-\frac{1}{12}(9+\sqrt{17})\right] \quad$ and $\quad\left[\frac{1}{12}(9+\sqrt{17}), \frac{4}{3}\right]$ and monotonously decreasing on the segments $\left[-\frac{1}{12}(9+\sqrt{17}),-\frac{2}{3}\right]$ and $\left[\frac{2}{3},-\frac{1}{12}(9+\sqrt{17})\right]$. This means that the extrema are at the points $-\frac{1}{12}(9+\sqrt{17})$ and $\frac{1}{12}(9+\sqrt{17})$ :

$$
\begin{aligned}
\sup _{x \in[-4 / 3,4 / 3]} g_{3}^{\prime}(x) & =g_{3}^{\prime}\left(-\frac{1}{12}(9+\sqrt{17})\right) \\
& =-g_{3}^{\prime}\left(\frac{1}{12}(9+\sqrt{17})\right) \\
& =\frac{(3+\sqrt{17}) \sqrt{7+\sqrt{17}}}{4 \sqrt{3}} .
\end{aligned}
$$

Since $g_{3}^{\prime}\left(-\frac{4}{3}\right)>0$ and $g_{3}^{\prime}\left(\frac{4}{3}\right)<0$, the total variation of $g_{3}^{\prime}$ is

$\mathbf{V}\left[g_{3}^{\prime}\right]=\mathbf{V}_{-\frac{4}{3}}^{\frac{4}{3}}\left[g_{3}^{\prime}\right]=4 \sup _{x \in[-4 / 3,4 / 3]} g_{3}^{\prime}(x)=\frac{(3+\sqrt{17}) \sqrt{7+\sqrt{17}}}{\sqrt{3}}$.

Then, using Proposition 2.2 [13], for $x \in(0,2 \pi]$ we get

$$
\begin{aligned}
\left|\varphi_{n}(x)\right|= & \frac{1}{2 \sqrt{N_{n}}}\left|\sum_{k \in \mathbb{Z}} g_{3}\left(\frac{k}{N_{n}}\right) \cos k x\right| \\
& \leq \frac{C}{\sqrt{N_{n}}|x|^{2}} \sum_{k \in \mathbb{Z}}\left|\Delta^{2} g_{3}\left(\frac{k}{N_{n}}\right)\right|,
\end{aligned}
$$

where $\Delta^{2} g_{3}\left(\frac{k}{N_{n}}\right)=g_{3}\left(\frac{k}{N_{n}}\right)-2 g_{3}\left(\frac{k+1}{N_{n}}\right)+g_{3}\left(\frac{k+2}{N_{n}}\right)$.

From the mean value theorem we can deduce that there exist points $m_{k} \in(k, k+1)$ such that

$$
\Delta^{2} g_{3}\left(\frac{k}{N_{n}}\right)=\frac{1}{N_{n}}\left(g_{3}^{\prime}\left(\frac{m_{k+1}}{N_{n}}\right)-g_{3}^{\prime}\left(\frac{m_{k}}{N_{n}}\right)\right) .
$$

Since the system of the points $\left\{\frac{m_{k}}{N_{n}}, k \in \mathbb{Z}\right\}$ is some partition of the real line and $g_{3}$ is a function having a first derivative of finite total variation $\mathbf{V}\left[g_{3}^{\prime}\right]$, it holds that

$$
\begin{aligned}
\sum_{k \in \mathbb{Z}}\left|\Delta^{2} g_{3}\left(\frac{k}{N_{n}}\right)\right|= & \sum_{k \in \mathbb{Z}} \frac{1}{N_{n}}\left|\left(g_{3}^{\prime}\left(\frac{m_{k+1}}{N_{n}}\right)-g_{3}^{\prime}\left(\frac{m_{k}}{N_{n}}\right)\right)\right| \\
& \leq \frac{1}{N_{n}} \mathbf{V}\left[g_{3}^{\prime}\right]=\frac{C}{2^{n}} .
\end{aligned}
$$


Thus, from (5.3)-(5.5) we get

$$
\left|\varphi_{n}(x)\right| \leq \frac{C}{2^{3 n / 2}|x|^{2}} .
$$

The function $\psi_{n}$ can be estimated in the same way. We have that

$$
\psi_{n}(\cdot)=\frac{1}{\sqrt{N_{n}}} \sum_{k \in \mathbb{Z}} g_{4}\left(\frac{k}{N_{n}}\right) \cos k \cdot
$$

where

$$
g_{4}(x)= \begin{cases}\frac{1+3(x-1)}{\sqrt{1+9(x-1)^{2}}}, & 1-\frac{1}{3} \leq x \leq 1+\frac{1}{3} \\ \frac{2-3(x-2)}{\sqrt{4+9(x-2)^{2}}}, & 1+\frac{1}{3}<x \leq 2+\frac{2}{3} \\ 0, & \text { else }\end{cases}
$$

is an aperiodic coefficient function.

From the first and second derivatives $g_{4}^{\prime}$ and $g_{4}^{\prime \prime}$, we get that the points $\frac{1}{6}(9+\sqrt{17})$ and $\frac{1}{12}(15-\sqrt{17})$ are extrema of the function $g_{4}^{\prime}$. Since $g_{4}^{\prime}\left(1-\frac{1}{3}\right)>0$ and $g_{4}^{\prime}\left(2+\frac{2}{3}\right)<0$, the total variation of $g_{4}^{\prime}$ is

$$
\begin{aligned}
\mathbf{V}\left[g_{4}^{\prime}\right] & =2 g_{4}^{\prime}\left(\frac{1}{12}(15-\sqrt{17})\right)-2 g_{4}^{\prime}\left(\frac{1}{6}(9+\sqrt{17})\right) \\
& =\frac{\sqrt{3}(\sqrt{17}+3) \sqrt{7+\sqrt{17}}}{4} .
\end{aligned}
$$

Using the same consideration as above, we have

$$
\left|\psi_{n}(x)\right| \leq \frac{C}{2^{3 n / 2}|x|^{2}} \mathbf{V}\left[g_{4}^{\prime}\right] \leq \frac{C}{2^{3 n / 2}|x|^{2}} .
$$

The main ingredient to prove the following lemmata is using estimations (5.1) and (5.2). Therefore, we formulate and prove these results for functions $\psi_{n}, n \in \mathbb{N}$, but they are true also for functions $\varphi_{n}, n \in \mathbb{N}$.

Further, by $I^{\prime}$ we denote the complement of the interval $I \subset$ $[0,2 \pi)$ to the segment $[0,2 \pi)$, i.e., $I^{\prime}:=[0,2 \pi) \backslash I$. In the case when $x_{0}=0$ we use corresponding modification: $I^{\prime}$ : = $[-\pi, \pi) \backslash I$, where $I \subset[-\pi, \pi)$.

Lemma 5.3. Let $I \subset \mathbb{R},|I|<2 \pi$, be an interval centered at $x_{0}, J$ be an interval centered at $x_{0}$ such that $J \subset I$, and $\zeta$ be an infinitely differentiable on $\mathbb{R}$ function supported on $J$ and extended $2 \pi$-periodically, and let $f \in L_{2 \pi}^{1}$. Then, for $1 \leq p<\infty$

$$
\begin{aligned}
& \left(\sum_{s=0}^{2 N_{n}-1}\left|\left((f \zeta) * \psi_{n}\right)\left(\frac{s \pi}{N_{n}}\right)\right|^{p}\right)^{\frac{1}{p}} \\
& \leq\left(\sum_{s \in \kappa(I, n)}\left|\left((f \zeta) * \psi_{n}\right)\left(\frac{s \pi}{N_{n}}\right)\right|^{p}\right)^{\frac{1}{p}}+C_{1}(I, \zeta, f) 2^{-n\left(\frac{3}{2}-\frac{1}{p}\right)},
\end{aligned}
$$

and for $p=\infty$

$$
\begin{aligned}
\max _{s=0, \ldots, 2 N_{n}-1}\left|\left((f \zeta) * \psi_{n}\right)\left(\frac{s \pi}{N_{n}}\right)\right| & \leq \max _{s \in \kappa(I, n)}\left|\left((f \zeta) * \psi_{n}\right)\left(\frac{s \pi}{N_{n}}\right)\right| \\
& +C_{2}(I, \zeta, f) 2^{-\frac{3 n}{2}} .
\end{aligned}
$$

Proof. Without loss of generality, we can assume that the interval $J$ has a length equal to $|I| / 2$. First, we consider the case $1 \leq p<$ $\infty$. Using the inequality $(a+b)^{q} \leq a^{q}+b^{q}, a, b>0,0<q \leq 1$, with $q=1 / p$, we have

$$
\begin{aligned}
& \left(\sum_{s=0}^{2 N_{n}-1}\left|\left((f \zeta) * \psi_{n}\right)\left(\frac{s \pi}{N_{n}}\right)\right|^{p}\right)^{\frac{1}{p}} \\
\leq & \left(\sum_{s \in \kappa(I, n)}\left|\left((f \zeta) * \psi_{n}\right)\left(\frac{s \pi}{N_{n}}\right)\right|^{p}\right)^{\frac{1}{p}} \\
+ & \left(\sum_{s \in \kappa(I I, n)}\left|\left((f \zeta) * \psi_{n}\right)\left(\frac{s \pi}{N_{n}}\right)\right|^{p}\right)^{\frac{1}{p}} .
\end{aligned}
$$

Let us estimate the second term in this inequality. From the inequality (4.11), for $1 \leq p<\infty$ we have

$$
\begin{aligned}
& \left(\sum_{s \in \kappa\left(I^{\prime}, n\right)}\left|\left((f \zeta) * \psi_{n}\right)\left(\frac{s \pi}{N_{n}}\right)\right|^{p}\right)^{1 / p} \\
& =\left(\left.\sum_{s \in \kappa\left(I^{\prime}, n\right)}\left|\frac{1}{2 \pi} \int_{0}^{2 \pi} f(x) \zeta(x) \psi_{n}\left(x-\frac{s \pi}{N_{n}}\right) \mathrm{d} x\right|^{p}\right|^{1 / p}\right. \\
& =\left(\left.\sum_{s \in \kappa\left(I^{\prime}, n\right)}\left|\frac{1}{2 \pi} \int_{J} f(x) \zeta(x) \psi_{n}\left(x-\frac{s \pi}{N_{n}}\right) \mathrm{d} x\right|^{p}\right|^{1 / p}\right. \\
& \leq \frac{1}{2 \pi} \int_{J}\left(\sum_{s \in \kappa\left(I^{\prime}, n\right)}|f(x)|^{p}|\zeta(x)|^{p}\left|\psi_{n}\left(x-\frac{s \pi}{N_{n}}\right)\right|^{p}\right)^{1 / p} \mathrm{~d} x .
\end{aligned}
$$

Since $x \in J$ and $\frac{\left(s+2 k N_{n}\right) \pi}{N_{n}} \in I^{\prime}$ for some $k \in \mathbb{Z}$, we have that $|I| / 4<\left|x-\frac{\left(s+2 k N_{n}\right) \pi}{N_{n}}\right|$. Using Lemma 5.2, we obtain

$$
\begin{aligned}
& \left(\sum_{s \in \kappa\left(I^{\prime}, n\right)}\left|\left((f \zeta) * \psi_{n}\right)\left(\frac{s \pi}{N_{n}}\right)\right|^{p}\right)^{1 / p} \\
& \left.\leq \frac{C}{2^{3 n / 2}} \int_{J} \sum_{s \in \kappa\left(I^{\prime}, n\right)}|f(x)|^{p}|\zeta(x)|^{p} \frac{1}{\left|x-\frac{\left(s+2 k N_{n}\right) \pi}{N_{n}}\right|^{2 p}}\right)^{1 / p} \mathrm{~d} x \\
& \leq \frac{C}{2^{3 n / 2}(|I| / 4)^{2}} \int_{J}\left(\sum_{s \in \kappa\left(I^{\prime}, n\right)}|f(x)|^{p}|\zeta(x)|^{p}\right)^{1 / p} \mathrm{~d} x \\
& \leq \frac{C(I)}{2^{n\left(\frac{3}{2}-\frac{1}{p}\right)}} \int_{J}|f(x)||\zeta(x)| \mathrm{d} x \leq \frac{C(I, \zeta, f)}{2^{n\left(\frac{3}{2}-\frac{1}{p}\right)}} .
\end{aligned}
$$


Let $p=\infty$. From the obvious inequality $\max _{s \in K}\left|a_{s}\right| \leq \max _{s \in K_{1}}\left|a_{s}\right|+$ $\max _{s \in K_{2}}\left|a_{s}\right|, K=K_{1} \cup K_{2}, K_{1} \cap K_{2}=\emptyset$, where $K$ is a finite set of indices, we get

$$
\begin{aligned}
\max _{s=0, \ldots, 2 N_{n}-1}\left|\left((f \zeta) * \psi_{n}\right)\left(\frac{s \pi}{N_{n}}\right)\right| & \leq \max _{s \in \kappa(I, n)}\left|\left((f \zeta) * \psi_{n}\right)\left(\frac{s \pi}{N_{n}}\right)\right| \\
& +\max _{s \in \kappa\left(I^{\prime}, n\right)}\left|\left((f \zeta) * \psi_{n}\right)\left(\frac{s \pi}{N_{n}}\right)\right|
\end{aligned}
$$

Let us estimate the second term in the last inequality. Using a similar consideration (with corresponding modification) as in the case $1 \leq p<\infty$, we have

$$
\begin{aligned}
& \max _{s \in \kappa\left(I^{\prime}, n\right)}\left|\left((f \zeta) * \psi_{n}\right)\left(\frac{s \pi}{N_{n}}\right)\right| \\
& \leq \max _{s \in \kappa\left(I^{\prime}, n\right)} \frac{1}{2 \pi} \int_{J}|f(x)||\zeta(x)|\left|\psi_{n}\left(x-\frac{s \pi}{N_{n}}\right)\right| \mathrm{d} x \\
& \leq \frac{C}{2^{3 n / 2}} \max _{s \in \kappa\left(I^{\prime}, n\right)} \int_{J}|f(x)||\zeta(x)| \frac{\mathrm{d} x}{\left|x-\frac{\left(s+2 k N_{n}\right) \pi}{N_{n}}\right|^{2}} \leq \frac{C(I, \zeta, f)}{2^{3 n / 2}} .
\end{aligned}
$$

Lemma 5.4. Let $I \subset \mathbb{R},|I|<2 \pi$, be an interval centered at $x_{0}$, $J_{1}$ and $J$ be intervals centered at $x_{0}$ such that $J \subset J_{1} \subset I$, and $\zeta$ be an infinitely differentiable on $\mathbb{R}$ function supported on $I$ and extended $2 \pi$-periodically such that $\zeta(x)=1$ for all $x \in J_{1}$, and let $f \in L_{2 \pi}^{1}$. Then, for $1 \leq p<\infty$, we have

$$
\begin{aligned}
& \left(\sum_{s \in \kappa(J, n)}\left|\left(f * \psi_{n}\right)\left(\frac{s \pi}{N_{n}}\right)\right|^{p}\right)^{\frac{1}{p}} \\
& \leq\left(\sum_{s \in \kappa(J, n)}\left|\left((f \zeta) * \psi_{n}\right)\left(\frac{s \pi}{N_{n}}\right)\right|^{p}\right)^{\frac{1}{p}}+C_{1}(I, \zeta, f) 2^{-n(3 / 2-1 / p)},
\end{aligned}
$$

and for $p=\infty$

$$
\begin{aligned}
\max _{s \in \kappa(J, n)}\left|\left(f * \psi_{n}\right)\left(\frac{s \pi}{N_{n}}\right)\right| & \leq \max _{s \in \kappa(J, n)}\left|\left((f \zeta) * \psi_{n}\right)\left(\frac{s \pi}{N_{n}}\right)\right| \\
& +C_{2}(I, \zeta, f) 2^{-\frac{3 n}{2}}
\end{aligned}
$$

Proof. Without loss of generality we can assume that $\left|J_{1}\right|=|I| / 2$ and $|J|=|I| / 4$. Applying Minkowski's inequality for sums (with corresponding modification for $p=\infty$ )

$$
\left(\sum_{k}\left|a_{k}+b_{k}\right|^{p}\right)^{\frac{1}{p}} \leq\left(\sum_{k}\left|a_{k}\right|^{p}\right)^{\frac{1}{p}}+\left(\sum_{k}\left|b_{k}\right|^{p}\right)^{\frac{1}{p}}
$$

we have that

$$
\begin{aligned}
& \left(\sum_{s \in \kappa(J, n)}\left|\left(f * \psi_{n}\right)\left(\frac{s \pi}{N_{n}}\right)\right|^{p}\right)^{1 / p} \\
\leq & \left(\sum_{s \in \kappa(J, n)}\left|\left((f \zeta) * \psi_{n}\right)\left(\frac{s \pi}{N_{n}}\right)\right|^{p}\right)^{1 / p} \\
& +\left(\sum_{s \in \kappa(J, n)}\left|\left((f(1-\zeta)) * \psi_{n}\right)\left(\frac{s \pi}{N_{n}}\right)\right|^{p}\right)^{1 / p} .
\end{aligned}
$$

Let us estimate the second term in this inequality. From inequality (4.11), for $1 \leq p<\infty$ we have

$$
\begin{aligned}
& \left(\sum_{s \in \kappa(J, n)}\left|\left((f(1-\zeta)) * \psi_{n}\right)\left(\frac{s \pi}{N_{n}}\right)\right|^{p}\right)^{1 / p} \\
& =\left(\sum_{s \in J_{n}}\left|\frac{1}{2 \pi} \int_{0}^{2 \pi} f(x)(1-\zeta(x)) \psi_{n}\left(x-\frac{s \pi}{N_{n}}\right) \mathrm{d} x\right|^{p}\right)^{1 / p} \\
& =\left(\sum_{s \in \kappa(J, n)}\left|\frac{1}{2 \pi} \int_{[0,2 \pi) \backslash J_{1}} f(x)(1-\zeta(x)) \psi_{n}\left(x-\frac{s \pi}{N_{n}}\right) \mathrm{d} x\right|^{p}\right)^{1 / p} \\
& \leq \frac{1}{2 \pi} \int_{[0,2 \pi) \bigcup_{1}}\left(\sum_{s \in \kappa(J, n)}|f(x)|^{p}|1-\zeta(x)|^{p}\left|\psi_{n}\left(x-\frac{s \pi}{N_{n}}\right)\right|^{p}\right)^{1 / p} \mathrm{~d} x .
\end{aligned}
$$

Since for $x \in[0,2 \pi) \backslash J_{1}$ and $\frac{\left(s+2 k N_{n}\right) \pi}{N_{n}} \in J$ for some $k \in \mathbb{Z}$, it holds that $|I| / 8<\left|x-\frac{\left(s+2 k N_{n}\right) \pi}{N_{n}}\right|$, from Lemma 5.2 we get

$$
\begin{aligned}
& \left(\sum_{s \in \kappa(J, n)}\left|\left((f(1-\zeta)) * \psi_{n}\right)\left(\frac{s \pi}{N_{n}}\right)\right|^{p}\right)^{1 / p} \\
& \leq \frac{C}{2^{3 n / 2}} \int_{[0,2 \pi) \backslash J_{1}}\left(\sum_{s \in \kappa(J, n)} \frac{|f(x)|^{p}|1-\zeta(x)|^{p}}{\left|x-\frac{\left(s+2 k N_{n}\right) \pi}{N_{n}}\right|^{2 p}}\right)^{1 / p} \mathrm{~d} x \\
& \leq \frac{C}{2^{3 n / 2}(|I| / 8)^{2}} \int_{[0,2 \pi) \backslash J_{1}}\left(\sum_{s \in \kappa(J, n)}|f(x)|^{p}|1-\zeta(x)|^{p}\right)^{1 / p} \mathrm{~d} x \\
& \leq \frac{C(I)}{2^{n\left(\frac{3}{2}-\frac{1}{p}\right)}} \int_{[0,2 \pi) \backslash J_{1}}|f(x)||1-\zeta(x)| \mathrm{d} x \leq \frac{C(I, \zeta, f)}{2^{n\left(\frac{3}{2}-\frac{1}{p}\right)}} .
\end{aligned}
$$

Using a similar consideration (with corresponding modification), for $p=\infty$ we have 


$$
\begin{aligned}
& \max _{s \in \kappa(J, n)}\left|\left((f(1-\zeta)) * \psi_{n}\right)\left(\frac{s \pi}{N_{n}}\right)\right| \\
& \leq \max _{s \in \kappa(J, n)} \frac{1}{2 \pi} \int_{[0,2 \pi) \backslash J_{1}}|f(x)||1-\zeta(x)|\left|\psi_{n}\left(x-\frac{s \pi}{N_{n}}\right)\right| \mathrm{d} x \\
& \leq \frac{C}{2^{3 n / 2}} \max _{s \in \kappa(J, n)} \int_{[0,2 \pi) \backslash V_{1}}|f(x)||1-\zeta(x)| \frac{\mathrm{d} x}{\left|x-\frac{\left(s+2 k N_{n}\right) \pi}{N_{n}}\right|^{2}} \\
& \leq \frac{C(I, \zeta, f)}{2^{3 n / 2}} .
\end{aligned}
$$

Proof of Theorem 3.2. The equivalence between (a) and (c) follows from Theorem 4.1 and the equivalence between (a) and (c) of Theorem 3.1.

Let now part (c) hold and $I$ be the interval chosen as in that part. Let $J_{1}$ and $J$ be intervals centered at $x_{0}$ such that $J \subset J_{1} \subset I$, and $\zeta$ be an infinitely differentiable on $\mathbb{R}$ function supported on $I$ and extended as a $2 \pi$-periodic function such that $\zeta(x)=1$ for all $x \in J_{1}$. Using Lemma 5.4 for $n \in \mathbb{N}$ and $1 \leq p \leq \infty$ (with corresponding modification for $p=\infty$ ), we get

$$
\begin{aligned}
& N_{n}^{1 / 2-1 / p}\left(\sum_{s \in \kappa(J, n)}\left|\left(f * \psi_{n}\right)\left(\frac{s \pi}{N_{n}}\right)\right|^{p}\right)^{\frac{1}{p}} \\
& \leq N_{n}^{1 / 2-1 / p}\left(\sum_{s \in \kappa(J, n)}\left|\left((f \zeta) * \psi_{n}\right)\left(\frac{s \pi}{N_{n}}\right)\right|^{p}\right)^{\frac{1}{p}}+C(I, \zeta, f) 2^{-n} \\
& \leq N_{n}^{1 / 2-1 / p}\left(\sum_{s=0}^{2 N_{n}-1}\left|\left((f \zeta) * \psi_{n}\right)\left(\frac{s \pi}{N_{n}}\right)\right|^{p}\right)^{\frac{1}{p}}+C(I, \zeta, f) 2^{-n}
\end{aligned}
$$

Since $\left\{2^{-n}\right\}_{n=0}^{\infty} \in b_{\theta, \alpha}$ for $0<\alpha<1$ and from the conditions of part (c), we have that part (b) also holds.

Let part (b) hold. Let $I$ be the interval as in that part and $I_{1}$ be an interval centered at $x_{0}$ such that $I_{1} \subset I$. Let $\zeta$ be an infinitely differentiable on $\mathbb{R}$ function supported on $I_{1}$ and extended $2 \pi$ periodically. Then, from Lemma 5.3 for $n \in \mathbb{N}$ and $1 \leq p \leq \infty$, we have

$$
N_{n}^{1 / 2-1 / p}\left(\sum_{s=0}^{2 N_{n}-1}\left|\left((f \zeta) * \psi_{n}\right)\left(\frac{s \pi}{N_{n}}\right)\right|^{p}\right)^{\frac{1}{p}}
$$

\section{REFERENCES}

1. Bazarkhanov DB. Wavelet approximation and Fourier widths of classes of periodic functions of several variables. I. Proc Steklov Inst Math. (2010) 269:2-24. translation from: Tr Mat Inst Steklova (2010) 269:8-30. doi: 10.1134/S0081543810020021

2. Dinh D. B-spline quasi-interpolant representations and sampling recovery of functions with mixed smoothness. J Complex. (2011) 27:541-67. doi: 10.1016/j.jco.2011.02.004

3. Hinrichs A, Markhasin L, Oettershagen J, Ullrich T. Optimal quasi-Monte Carlo rules on higher order digital nets for the numerical integration of multivariate periodic functions.

$$
\begin{aligned}
& \leq N_{n}^{1 / 2-1 / p}\left(\sum_{s \in \kappa(I, n)}\left|\left((f \zeta) * \psi_{n}\right)\left(\frac{s \pi}{N_{n}}\right)\right|^{p}\right)^{\frac{1}{p}} \\
& +C(I, \zeta, f) 2^{-n} \leq N_{n}^{1 / 2-1 / p}\left(\sum_{s \in \kappa(I, n)}\left|\left(f * \psi_{n}\right)\left(\frac{s \pi}{N_{n}}\right)\right|^{p}\right)^{\frac{1}{p}} \\
& +C(I, \zeta, f) 2^{-n} .
\end{aligned}
$$

Since $\left\{2^{-n}\right\}_{n=0}^{\infty} \in b_{\theta, \alpha}$ for $0<\alpha<1$ and from the conditions of part (b) we have that part (c) also holds.

Note that Theorem 3.1 and Theorem 3.2 were proved for the smoothness parameter $\alpha$ which takes values from the interval $(0,1)$. The proofs depends on the smoothness properties of the coefficient functions $g_{1}$ and $g_{2}$ (see (2.1) and (2.2)). One can prove these theorems for other values of the parameter $\alpha$ by taking "smoother" coefficient functions (2.7) and (2.8).

\subsection{Proof of Theorem 3.3}

The equivalence between parts (a) and (b) follows from Lemma 2.1 and the equivalence between parts (a) and (c) of Theorem A. To get equivalence between parts (b) and (c) we use Theorem 4.1 and formulas (2.7) and (2.8).

\section{AUTHOR CONTRIBUTIONS}

All authors listed, have made substantial, direct and intellectual contribution to the work, and approved it for publication.

\section{ACKNOWLEDGMENTS}

The authors were supported by FP7-People-2011-IRSES Project number 295164 (EUMLS: EU-Ukrainian Mathematicians for Life Sciences) and H2020-MSCA-RISE-2014 Project number 645672 (AMMODIT: Approximation Methods for Molecular Modeling and Diagnosis Tools). The authors would like to thank Viktor Romanyuk (Institute of Mathematics of NAS of Ukraine) for many valuable scientific discussions. Moreover, the authors would like to thank the referees for their helpful remarks and corrections.

Num Math. (2016) 134:163-96. doi: 10.1007/s00211-0150765-y

4. Kashin BS, Saakyan AA. Orthogonal Series. Translations of Mathematical Monographs, V.75, Providence: American Mathematical Society (1989).

5. Romanyuk VS. Multiple Haar basis and $m$-term approximation for functions from Besov classes. I. Ukr Math J. (2016) 68:625-37. translation from: Ukr Mat Zh. (2016) 68:551-62. doi: 10.1007/s11253-016-1246-x

6. Mhaskar HN, Prestin J. On local smoothness classes of periodic functions. J Fourier Anal Appl. (2005) 11:353-73. doi: 10.1007/s00041-005-4006-0

7. Prestin J, Selig KK. On a constructive representation of an orthogonal trigonometric Schauder basis for $C_{2 \pi}$. Oper Theory Adv Appl. (2001) 121:402-25. doi: 10.1007/978-3-0348-8276-7_22 
8. Lorentz R, Saakyan A. Orthonormal trigonometric Schauder bases of optimal degree for $C(K)$. J. Fourier Anal Appl. (1994) 1:103-12. doi: 10.1007/s00041-001-4005-8

9. Selig KK. Periodische Wavelet-Packets und Eine Gradoptimale schauderbasis. Shaker Verlag, Aachen: Thesis, Univ. Rostock 1997 (1998).

10. DeVore RA, Lorentz GG. Constructive Approximation. Berlin: Springer Verlag (1993).

11. Nikolskii SM. Approximation of Functions of Several Variables and Imbedding Theorems. Berlin: Springer (1975).

12. Dzyadyk VK, Shevchuk IA. Theory of Uniform Approximation of Functions by Polynomials. Berlin: Walter de Gruyter (2008).

13. Mhaskar HN, Prestin J. On the detection of singularities of a periodic function. Adv Comput Math. (2000) 12:95-131. doi: 10.1023/ A:1018921319865
14. Zygmund A. Trigonometric Series. Volume I. Cambridge: Cambridge University Press (1968).

15. Timan AF. Theory of Approximation of Functions of a Real Variable. Oxford: Pergamon Press (1963).

Conflict of Interest Statement: The authors declare that the research was conducted in the absence of any commercial or financial relationships that could be construed as a potential conflict of interest.

Copyright (c) 2017 Derevianko, Myroniuk and Prestin. This is an open-access article distributed under the terms of the Creative Commons Attribution License (CC BY). The use, distribution or reproduction in other forums is permitted, provided the original author(s) or licensor are credited and that the original publication in this journal is cited, in accordance with accepted academic practice. No use, distribution or reproduction is permitted which does not comply with these terms. 